\title{
Anesthetic Management for an Eye Surgery in a Patient with Stevens-Johnson Syndrome
}

\author{
Woo-Jong Shin, Hyung Joon Park, Chang Wook Lee, Seong Ho Park, Youngsuk Shon, Jong Hoon Yeom \\ Department of Anesthesiology and Pain Medicine, Hanyang University School of Medicine, Seoul, Korea
}

\begin{abstract}
The Stevens-Johnson syndrome is a severe manifestation of erythema multiforme associated with multisystem involvement. It is extremely unusual for patients with this syndrome to present with indications for eye surgery. Respiratory tract involvement can make the management of the airway and tracheal intubation difficult. We describe herein the perioperative management of a patient with this disease who underwent surgery for amniotic membrane transplantation.
\end{abstract}

Keywords: Anesthesia; Erythema multiforme; Stevens-Johnson syndrome

\section{INTRODUCTION}

Stevens-Johnson syndrome is a relatively rare disease that causes systemic symptoms, such as acute rash blisters in the skin and mucous membranes, and that can lead to death with pure skin disease. This syndrome is a combination of several symptoms, and the pattern varies from person to person. Skin symptoms include inflammation, macule, papules, vesicles, and extensive epidermal necrosis of the epidermis, with a high likelihood of secondary skin infections. Lesions can occur systemically, including the eyes, kidneys, and digestive system. Ulcers in the respiratory tract are common, and purulent sputum can be observed [1].

Stevens-Johnson syndrome is rarely an indication of surgery that requires general anesthesia with the onset. Endotracheal intubation may be difficult during general anesthesia due to buccal or pharyngeal blisters, erosions, and ulcers. Extensive epidermal necrosis and detachment can make it difficult to secure and maintain endotracheal tubes and monitoring devices. Consideration should be given to the loss of fluids and imbalances of electrolytes from skin damage, and systemic complications such as kidneys, heart, and respiratory tract.

We report a case of patients who underwent general anesthesia for amniotic membrane transplantation with conjunctival syn- echiae during inpatient treatment with Stevens-Johnson syndrome in the eye and face.

\section{CASE REPORT}

The patient is a 49-year-old woman, $154 \mathrm{~cm}$ tall and weighs $47 \mathrm{~kg}$, who has given antibiotics (quinolone) 15 days ago for acute frontal sinusitis. The patient visited the emergency room of Hanyang University Guri Hospital complaining of systemic muscle pain, weakness, angioedema around the lips, multiple skin rashes, and blisters on the face. Her body temperature was normal at the time of admission to the hospital, but she was hospitalized with extensive epidermal dropouts, edema and pain in the face and torso, eyelid swelling, conjunctival hyperemia, inflammatory blisters and ulcers around the mouth, erosion, ulcers, and bleeding in the mouth. Systemic steroids, antihistamines, and acetaminophen were administered for treatment. After 5 days of hospitalization, conjunctival adhesion developed. Conservative treatments were conducted for about a week, but eye lesions and pain did not improve. And due to the possibility of blindness, it was decided to perform an emergency amniotic membrane transplantation. Photographic data were not obtained because we didn't get the patient's consent.

Preoperative blood test, biochemical test, blood coagulation
Correspondence to: Woo-Jong Shin

Department of Anesthesiology and Pain Medicine, Hanyang University Guri Hospital, 153 Gyeongchun-ro, Guri 11923, Korea

Tel: +82-31-560-2390, Fax: +82-31-563-1731, E-mail: swj0208@hanyang.ac.kr

Received: Nov. 28, 2019/Accepted after revision: Dec. 11, 2019
(C) 2019 Soonchunhyang Medical Research Institute This is an Open Access article distributed under the terms of the Creative Commons Attribution Non-Commercial License (http://creativecommons.org/licenses/by-nc/4.0/). 
test, and arterial blood gas analysis were in a normal range, and body temperature was normal. In chest radiographs and computed tomography, no active lesions were observed except for suspected inactive pulmonary tuberculosis and bullae in both upper lobes. When entering the operating room, desquamation and bleeding of the whole face, inflammatory blisters and ulcers around the lips, and erosion, ulceration, and bleeding of the oral cavity were observed, but the consciousness was clear and other symptoms such as dyspnea were not complained.

The patient was moved to the surgery table, paying attention to additional damage to the skin lesion, and the thermometer was placed on the axilla, the normal skin area, and the electrocardiogram (ECG) pad was also attached to the dorsal area, the normal skin area. To prevent further damage caused by constant and repeated pressure and shearing forces in certain areas, the upper arm was first wrapped with a gauze pad, and then the blood pressure cuff was wrapped. Surgical pads were placed below the elbows and the heels to prevent pressure damage. The normal respiratory sound was observed in both lungs, and the arterial blood gas analysis showed $\mathrm{pH}$ 7.49, $\mathrm{PaCO}_{2} 25 \mathrm{~mm} \mathrm{Hg}, \mathrm{PaO}_{2} 104 \mathrm{~mm} \mathrm{Hg}$, base excess -3.2 mEq/L, hemoglobin $13.4 \mathrm{~g} / \mathrm{dL}$, and $\mathrm{Na}+/ \mathrm{K}+135 / 4.18 \mathrm{mEq} / \mathrm{L}$. Before preoxygenation, bleeding in the oral cavity was checked, and care was taken to remove as much as possible and to prevent further bleeding and damage. Due to the risk of epidermal detachment during manual ventilation, the mask was kept as close to the face as possible, encouraged deep breathing, so that preoxygenation was sufficiently achieved. Anesthesia and muscle relaxation were induced with penthotal sodium $175 \mathrm{mg}$, fentanyl $50 \mu \mathrm{g}$, and rocuronium bromide $30 \mathrm{mg}$, and the mask was gently adhered to the face. After manual ventilation, an internal diameter of $7.0 \mathrm{~mm}$ endotracheal tube was intubated without difficulty. The endotracheal tube was fixed to the maxillary incisor with a wire in consideration of the lesion around the mouth. Anesthesia was maintained with $50 \% \mathrm{~N}_{2} \mathrm{O} / \mathrm{O}_{2}$ and $5 \mathrm{vol} \%$ of desflurane and $0.06 \mu \mathrm{g} / \mathrm{kg} / \mathrm{min}$ remifentanil. Sufficient fluids were administered to compensate for fluid loss from skin lesions, and urinary catheterization was not done due to concern for urinary tract infection. Because oral steroids were administered preoperatively, $100 \mathrm{mg}$ of hydrocortisone sodium was administered intravenously during anesthesia. Vital signs during surgery and anesthesia remained normal. Surgery and anesthesia took 3 hours and 45 minutes, and 100\% oxygen was supplied after the muscle relaxation reversal drug. After the patient's airway reflex and self breathing returned, extubation was done with caution against oral lesions. After moving to the postanesthesia care unit, no oral and intranasal active bleeding was observed, and no additional lesions were observed on the skin lesions. The patient was transferred to the general ward with 8 points of postanesthetic recovery score. After that, both skin and ophthalmic lesions were recovered and discharged.

\section{DISCUSSION}

Stevens-Johnson syndrome is the most severe form of erythema multiforme and very rare. It is common in children under 30 years of age, with a high incidence in winter, with a mortality rate of $6 \%-$ $25 \%[2,3]$. Various causes such as infections caused by viruses, bacteria, and hypersensitivity reactions to drugs have been suggested as triggers, but have not been accurately identified [4]. The incidence is unknown, but it can occur at all ages, especially in children than in adults, with mortality rates of $3 \%-18 \%$ in case of children $[1,5]$. After a long clinical course of 2-7 weeks, relapse rates are reported by up to $20 \%$. Prodromal symptoms include high fever, boredom, cough, pain in the throat and mouth, chest pain, vomiting, diarrhea, myalgia, and arthralgia, followed by sudden inflammatory blister lesions of the skin and mucous membranes. Cutaneous lesions progress with epidermal detachment and are accompanied by ulceration of the mucous membranes of the nasal cavity, oral cavity, larynx, airway, and bronchus [4-8]. Complications include conjunctivitis, eyelid inflammation, corneal ulceration, anterior uveitis, panophthalmitis, corneal opacities, synechiae, and blindness $[2,6]$. Respiratory involvement is manifested in a variety of ways, with bronchial pneumonia being common and bilateral lung infiltration on chest radiographs [9]. Bullae of visceral pleura may result in bilateral pneumothorax accompanied by bronchopleural fistulas and subcutaneous emphysemas. Rarely, atrial fibrillation and myocarditis are reported [4-7].

Cautions for anesthesia management can be divided into premedication, patient movement, airway management, intravenous access, the balance of fluid and electrolyte, and fixation of monitoring devices. Care should be taken when moving to the operating room, and pre-anesthesia medications should be kept to a minimum so that the patient can move and maintain his or her position $[1,6]$. The patient in the case report did not perform pre-anesthesia medication in consideration of the patient's condition such as underlying lung disease and oral lesions and underwent emergency surgery. Patients receiving oral steroids before surgery need 
steroid administration [1]. Endotracheal intubation can be made more difficult by rupturing hemorrhagic blisters when applied with facial masks, oropharyngeal and nasopharyngeal airways, endotracheal tubes, esophageal stethoscopes, or temperature sensors, resulting in aspiration of tissue debris in the respiratory tract. Intraoral aspiration should be done carefully and kept to a minimum as possible. Facial masks may be covered with soft cotton wool coated with $0.5 \%$ hydrocortisone cream or gel. One-step smaller internal diameter endotracheal tube is used to minimize contact of the laryngeal inlet. The patient of this case had epidermal detachment and blister of the facial, ocular, and upper body. Because of intraoral erosion, ulceration, and bleeding, care was taken to minimize damage to the skin, mouth, and pharynx during intubation and fixation. Positive ventilation should be avoided if possible because of the possibility of pneumothorax caused by the rupture of the bullae. Intravenous catheterization can cause delayed skin healing and secondary infection. Urinary catheterization could lead to urethritis [7]. Attachment and maintenance of monitoring devices such as blood pressure monitors and electrocardiograms can exacerbate epidermal detachment and bleeding from ulcers, thus placing a pad under the blood pressure monitor to reduce the shear force of the upper and lower skin. It has been reported that it is safe to maintain continuous intra-arterial blood pressure monitoring in case of long operation time or frequent blood pressure measurement is needed [1,7], but this case was not performed. Because of the potential for myocarditis and arrhythmia, 5-lead electrocardiogram monitoring is recommended, and ECG pads should be used for non-adhesive or intradermal needle electrodes to attenuate skin lesions exacerbation [6,7].

In addition, to protect the eye, cover it with sterile ointment instead of gauze. Make sure the patient's seat is not wrinkled, and place the pads under the elbows and heels. Fever, upper respiratory tract infections, and pneumonia are common, and for this reason, do not stop or postpone diagnostic or therapeutic interventions that affect the prognosis [10].

If the fever occurs frequently, consider using a cooling blanket, etc., and measuring the temperature should be done in the axilla, where the lesion rarely invades. Commonly used intravenous an- esthetics include barbiturates, narcotics, and ketamine. Thiopental and methohexital should not be used because they cause or worsen the syndrome. Narcotics should not be administered unless proper airway maintenance is in place. Ketamine is an ideal anesthetic that can be safely used in patients with difficult airway maintenance because it maintains rapid anesthesia and laryngeal reflexes without a face mask and airway maintainer [7,10]. It has the advantage of being a powerful analgesic, so even at low doses, it can produce excellent analgesic and sedative effect. It also plays a role in systemic blood pressure maintenance and protection from pulmonary edema caused by sepsis [10].

Stevens-Johnson syndrome is a very rare and extensive mucosal skin disease that is a high risk of general anesthesia due to invasion of the entire respiratory system and pleura.

We experienced a case of general anesthesia for ophthalmic surgery in Stevens-Johnson syndrome. We need to identify the site of involvement before anesthesia, select a suitable drug, and prevent further damage to the lesion. We report with a literature review because we should fully recognize and pay close attention to the various complications that may arise.

\section{REFERENCES}

1. Smith GB, Shribman AJ. Anaesthesia and severe skin disease. Anaesthesia 1984;39:443-55.

2. Ting HC, Adam BA. Stevens-Johnson syndrome: a review of 34 cases. Int J Dermatol 1985;24:587-91.

3. Araujo OE, Flowers FP. Stevens-Johnson syndrome. J Emerg Med 1984;2: 129-35.

4. Schartum S. Stevens-Johnson syndrome with cardiac involvement: report of two cases. Acta Med Scand 1966;179:729-35.

5. Cucchiara RF, Dawson B. Anesthesia in Stevens-Johnson syndrome: report of a case. Anesthesiology 1971;35:537-9.

6. Benumof JL. Anesthesia and uncommon diseases. In: Benumof JL, editor. Anesthesia and uncommon diseases. 4th ed. Philadelphia (PA): W.B. Saunders Company; 1998. p. 428-31.

7. Kalhan SB, Ditto SR. Anesthetic management of a child with StevensJohnson syndrome. Cleve Clin J Med 1988;55:467-9.

8. Nazif MM, Ranalli DN. Stevens-Johnson syndrome: a report of fourteen pediatric cases. Oral Surg Oral Med Oral Pathol 1982;53:263-6.

9. Chanda JJ, Callen JP. Erythema multiforme and the Stevens-Johnson syndrome. South Med J 1978;71:566-70.

10. Madan R, Chawla R, Dhar P, Saxena A, Dada VK. Anesthesia in Stevens Johnson syndrome. Indian Pediatr 1989;26:1038-40. 